\title{
Untersuchung eines Prozessroboters als Messroboter in der Montage
}

\author{
Prof. Dr.-Ing. Rainer Müller ${ }^{1,2}$, Dr.-Ing. Matthias Scholer ${ }^{2}$, Ali Kanso M.Sc. ${ }^{2}$, Anne Blum M.Sc. ${ }^{1}$ \\ ${ }^{1}$ Universität des Saarlandes, Lehrstuhl für Montagesysteme, Eschberger Weg 46, 66121 Saarbrücken, \\ Deutschland \\ 2ZeMA - Zentrum für Mechatronik und Automatisierungstechnik gemeinnützige GmbH, Gruppe \\ Montageverfahren und -automatisierung, Eschberger Weg 46, 66121 Saarbrücken, Deutschland
}

\section{Zusammenfassung}

Im Zuge der Produktionsdigitalisierung werden mehr Daten aufgenommen und ausgewertet. Neue Roboter besitzen eine integrierte Drehmomentensensorik. Diese erfasst nicht nur interne Roboterdaten, sondern auch externe Einflüsse. Diese Daten können zur Inbetriebnahme von Prozessen oder zur Aussage der Qualität verwendet werden. Prozesse werden somit transparenter und quantifizierbarer. Um den Roboter als Messgerät einzubinden, bedarf es einer Untersuchung der Prüfprozesseignung. Zur Untersuchung wurde eine neuartige Vorgehensweise entwickelt, welche zwei vorhandene Methoden aus unterschiedlichen Disziplinen miteinander kombiniert. Zum einen wird die Methode DMAIC aus dem Qualitätsmanagement zur Strukturierung der Untersuchung und zum anderen wird der Eignungsnachweis von Prüfprozessen zur inhaltlichen Ermittlung von Wissen verwendet. Durch einen Versuchsaufbau werden Ergebnisse identifiziert, um die systematische und zufällige Messabweichung des Roboters zu bestimmen. Ziel dieses Papers ist die Analyse für den Einsatz eines Roboters als Messgerät im Montageprozess um somit neue Anwendungsfelder zu ermöglichen.

Keywords: Roboter, Drehmomentensensor, Montage, Messunsicherheit

\section{Einleitung und Motivation}

In der Produktion nimmt die Anzahl der Prüfprozesse durch steigende Qualitätsanforderungen stetig zu. Ziel ist Wissen über das Produkt und den Prozess zu generieren. Nach der Gesellschaft für Messund Automatisierungstechnik (GMA) des VDI/VDE ergeben sich daraus neue Anforderungen für die Messtechnik: „Schneller, Sicherer, Genauer und Flexibler" [1]. Um den Anforderungen gerecht zu werden, gibt es seit Jahren einen Trend zur Integration der Messsysteme in die Produktion. Messungen finden nicht mehr im separaten Messraum, sondern in der Montagelinie statt. Durch die Integration wird eine 100\%-Prüfung der Produkte ermöglicht. Einerseits sind die Auswahl und das Anwendungsspektrum von Messsystemen enorm, andererseits wird in vielen Unternehmen die Messtechnik als ein reiner Kostenfaktor gesehen. [1] Bei dem Einsatz in der Produktion ist zusätzlich ein Prüfmittelmanagement notwendig. Dieses sorgt dafür, dass das richtige Prüfmittel zum richtigen Zeitpunkt, am richtigen Ort, in einem einsatzbereiten Zustand zur Verfügung steht [2]. Dies ist mit zusätzlichen Kosten, Zeit und Ressourcen verbunden. Gleichzeitig schränkt es die Flexibilität ein, welche heutzutage immer bedeutender wird.

Im Zuge von Industrie 4.0 werden für eine angepasste Automatisierung Mensch-RoboterKooperationen (MRK) in der Montage eingesetzt. Die verwendeten Leichtbauroboter besitzen eine integrierte Sensorik, wie z.B. Drehmomentensensoren in den Roboterachsen. Diese Sensoren dienen zum Schutz, um Kollisionen rechtzeitig zu erkennen und externe Drehmomente zu ermitteln. Das Lean Management handelt von der Vermeidung von Verschwendung in der Produktion. Prozesse sollen schlank, beispielsweise ohne Wartezeit, sinnlose Transporte oder Nacharbeit erfolgen. [3] Beeinflusst durch diesen Gedanken, d.h. der konstanten Verbesserung, ist es sinnvoll den vorhandenen Prozessroboter auch gleichzeitig als Messgerät zu nutzen. Während des eigentlichen Montageprozesses kann so parallel eine In-Prozess-Messung erfolgen. Anhand der gemessenen 
Drehmomente in den Roboterachsen können die Masse und der Massenschwerpunkt eines Roboterwerkzeuges sowie von einem zu greifenden Bauteil im Montageprozess bestimmt werden. Dieser Ansatz spart Ressourcen sowie Kosten und steigert die Wandlungsfähigkeit im Prozess, da keine zusätzlichen Betriebsmittel implementiert werden müssen. Um diese Idee in der Praxis umzusetzen, bedarf es einer ausführlichen Betrachtung des Messroboters, um herauszustellen wie zuverlässig dessen Messergebnisse sind.

\section{Stand der Technik}

\section{Prüfprozesseignung}

Laut der GUM (Guide to the Expression of Uncertainly in Measurement) existiert ein wahrer Wert der Messgröße. Grundsätzlich ist dieser unbekannt. Die Messunsicherheit ist ein Maß dafür, wie gut der Mensch glaubt, den wahren Wert zu kennen. [4] Es gibt zwei Arten von Messabweichungen, die systematischen und die zufälligen. Die systematische Messabweichung ist die Differenz (Offset) zwischen dem Referenzwert eines Normals und dem Mittelwert der gemessenen Werte. Beschrieben wird sie durch den sogenannten Bias. Durch nicht beherrschte Einflussgrößen erfolgt bei einer wiederholten Messung eine andere Messwertausgabe. Diese zufällige Messabweichung lässt sich nicht vorhersehen und wird durch die Standardabweichung beschrieben. [5,6,7]

Zur Feststellung der Messunsicherheit gibt es standardisierte Vorgehensweisen. Dazu zählen Unternehmensrichtlinien, wie von Bosch oder Ford, die Measurement System Analysis (MSA) von der Automotive Industry Action Group (AIAG) und der Verband der Deutschen Automobilindustrie (VDA) mit dem Band 5 Prüfprozesseignung $[6,7]$. Die Vorgehensweise zur Beurteilung eines Messsystems ist bei allen
Verfahren ähnlich. Unter realen Bedingungen erfolgen Messungen. Die gewonnenen Messwerte werden grafisch und numerisch analysiert und Kennwerte bestimmt, welche mit vorgegebenen Grenzwerten verglichen werden. Der Unterschied der Verfahren liegt in der Berechnung der Kennwerte sowie der Betrachtung der Anzahl der Einflussgrößen. [8] Die MSA hat einen hohen Bekanntheitsgrad und ist Basis für viele Firmenrichtlinien. In der MSA erfolgt die Berechnung der GRR-Wertes (GageRepeatility and Reproducibility). Die Firmenrichtlinien erweitern dies durch die Bestimmung der Cg- bzw. Cgk-Werte, welche die systematische Messabweichung untersucht (Verfahren 1). Der VDA Band 5 hingegen basiert auf der ISO-Norm sowie der GUM. Das Beurteilungskriterium ist ein Eignungskennwert QMP (Capability Ratio Process), der die doppelte erweiterte Messunsicherheit ins Verhältnis zur Toleranz setzt. [8]

\section{Messungen in der Montage}

In der VDI/VDE erfolgt die Unterteilung nach dem Grad der Integration in die Produktion [1]. Die Unterteilung kann in diesem Fall, wie in Abb. 1 dargestellt auf die Montage übertragen werden. Messungen können in einem separaten Messraum (Montagefern), in der Umgebung der Montagelinie (Montagenah), in einer Messstation der Montagelinie (Inline), vor oder nach der Montage (Pre- bzw. PostProzess) oder während der eigentlichen Montage (In-Prozess) erfolgen. Durch die zunehmende Integration in die Montage sinkt die Reaktionszeit in den Prozess einzugreifen. Mit der In-Prozess-Messung erfolgt eine 100\% Prüfung. Einerseits werden so keine fehlerhaften Produkte an den Kunden weitergegeben, was zugleich einer Null-FehlerStrategie entspricht [9]. Andererseits steigen die Einfluss- bzw. Störgrößen auf die eigentliche Messung. In der Montage herrschen nicht immer konstante Bedingungen, so dass Störgrößen wie beispielsweise die Umwelt berücksichtigt werden müssen.

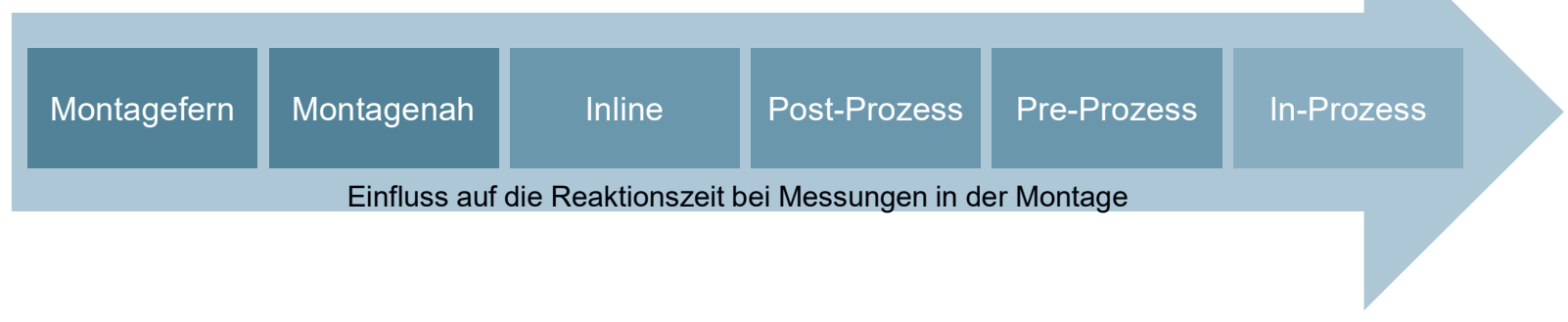

Abb. 1: $\quad$ Integrationsgrad der Messung in der Montage (in Anlehnung an [1]) 


\section{Roboter}

Zur Erprobung der Forschungsidee und Validierung der entwickelten Vorgehensweise wurde ein möglichst geeigneter Roboter ausgewählt.

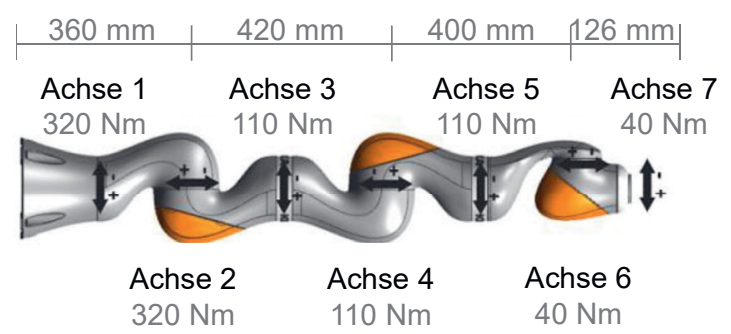

Abb. 2: Roboter KUKA LBR iiwa 14 R820 mit sieben Drehmomentensensoren

Der Roboter KUKA LBR iiwa 14 R820 ist ein Leichtbauroboter (LBR) und ein „intelligent industrial work assistant" (iiwa). Mit einer Traglast von $14 \mathrm{~kg}$ und einer Reichweite von $820 \mathrm{~mm}$ eignet er sich besonders für sensitive Fügeaufgaben sowie die Zusammenarbeit mit dem Menschen. Ermöglicht wird dies durch die besondere interne Sensorik und die Regelmechanismen. Dafür besitzt der LBR iiwa in allen Achsen Winkel- und Gelenkmomentensensoren. Neben der Kollisionserkennung kann die Sensorik auch für weitere Anwendungen verwendet werden. Greift der Roboter ein Werkstück, übt dies eine zusätzliche Kraft aus und hat somit Einfluss auf die gemessenen Momente in den Gelenkmomentensensoren. Der Roboter prüft den ermittelten Wert aus der Gelenkmomentensensor mit dem erwartetenen externen Drehmoment, das für eine Achse aufgrund des Robotermodells und der angegebenen Lastdaten berechnet wird. Die Drehmomentensensoren für den LBR iiwa 14 sind, wie in Abb. 2 dargestellt, unterschiedlich festgesetzt: $320 \mathrm{Nm}$ in Achse 1 und 2, $176 \mathrm{Nm}$ in Achse 3 und 4, $110 \mathrm{Nm}$ in Achse 5 sowie 40
$\mathrm{Nm}$ in Achse 6 und 7. Die achsspezifische Momentengenauigkeit wird mit $\pm 2 \%$ angegeben. [10]

\section{Methodische Vorgehensweise}

Ziel dieses Papers ist die Analyse für den Einsatz eines Roboters als Messgerät im Montageprozess. Dazu zählt eine erste Voruntersuchung wie genau die gemessenen Drehmomente sind. Für die Vorgehensweise werden zwei bestehende Ansätze aus der Literatur kombiniert. Die Methode DMAIC (Define, Measure, Analyse, Improve, Control) aus dem Qualitätsmanagementansatz Six Sigma wird verwendet [11]. DMAIC ist in der Produktion eine etablierte Methodik, vor allem wird sie in Kontinuierlichen Verbesserungsprozessen (KVP) eingesetzt. Die einzelnen DMAIC-Phasen dienen dem Prozessablauf der Untersuchung. Die Innovation ist, dass diese Phasen inhaltlich mit den Methoden der Prüfprozesseignung kombiniert werden. Durch die Verbindung der zwei Methoden, kann ein korrekter Ablauf der Untersuchung erfolgen. Die entwickelte Vorgehensweise ist schematisch in Abb. 3 dargestellt, beginnend mit der Beschreibung der Zielstellung, der Angabe zum Messsystem sowie einer Versuchsplanung. Anschließend folgt die Messdurchführung, Analyse der Messwerte sowie das Generieren von Verbesserungsideen für den Versuchsaufbau oder für spätere Applikationen, die Dokumentation sowie Kontrolle. Die Messungen am Roboter sowie die Analyse der gewonnenen Daten, geben Rückschlüsse auf die Streuung des Drehmomentes und auf die Wiederholbarkeit in den einzelnen Achsen. Dabei wird die Messunsicherheit des Roboters mit integrierten Drehmomentensensoren bestimmt. Bei der allgemeinen Prüfprozesseignung liegt ein Anwendungsfall mit bestimmten Toleranzangaben für ein

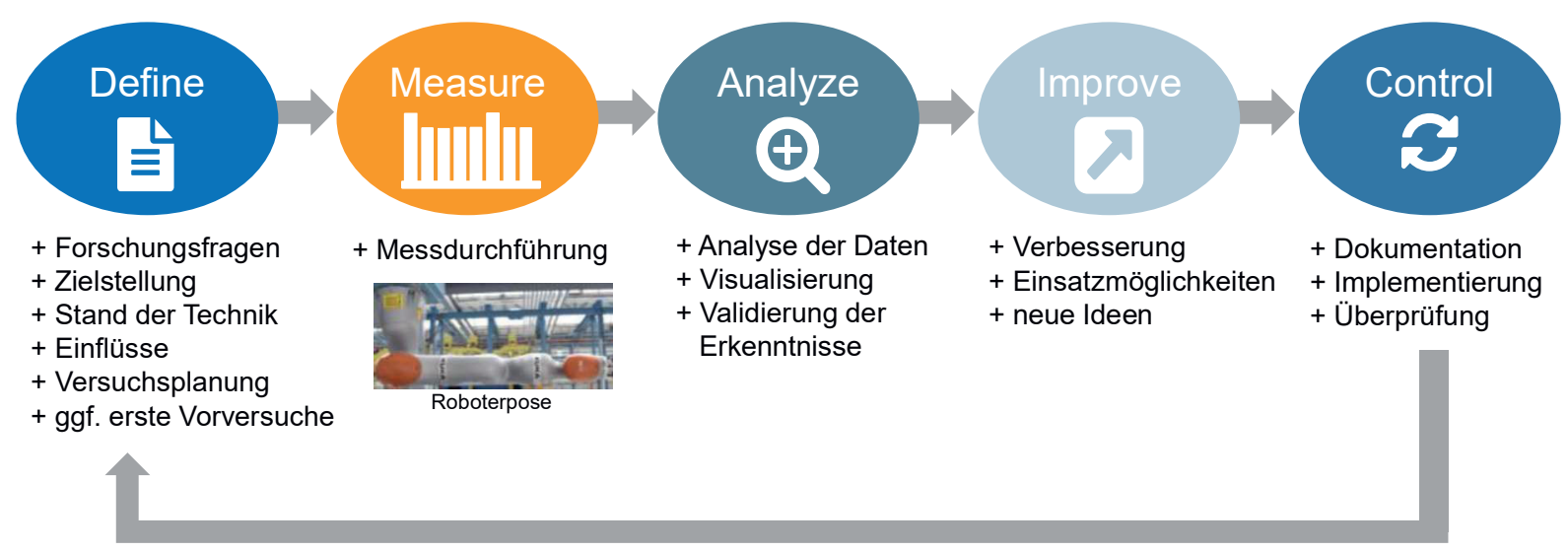

Abb. 3: Vorgehensweise der Untersuchung eines Prozessroboters als Messroboter 
Produktmerkmal vor. Eine Toleranzgrenze ist in diesem Fall nicht gegeben. Aus diesem Grund erfolgen Messungen mit Lasten über die gesamte Nutzlast am Roboterflansch, um dabei die Drehmomente in den Roboterachen zu beobachten und ein aussagekräftiges Ergebnisse zu erhalten.

\section{Umsetzung und Validierung}

In der Define-Phase wird die Zielstellung, Einflussmöglichkeiten auf die Messung und Messplanung definiert. Der Versuchsaufbau wird mit möglichst geringen Einflussgrößen gewählt. Durch Lasten werden Drehmomente erzeugt, aufgenommen und analysiert. Dazu werden am Roboterflansch Gewichte der gesamten Nutzlast, d.h. von wenigen Gramm bis zur Nennlast befestigt um eine vollständige Aussagekraft zu erhalten. Das Drehmoment in den Achsen wird während der Messungen aufgezeichnet und kann im Anschluss ausgewertet werden. Der Roboter befindet sich während der Messung in einer definierten Pose, d.h. Achse 2 bei 90 Grad und die restlichen Achsen bei 0 Grad (siehe Abb. 3).

Da keine Messung exkat ist, sondern das Ergebnis vom Messsystem, dem Messverfahren, der Qualifikation des Bedieners, der Umgebung und anderen Einflüssen abhängt, werden diese Einflüsse für den Versuchsaufbau dokumentiert [5,6]. Ein Ishikawa-Diagramm in Abb. 4 zeigt die Einflusskomponenten auf das Messystem. Es ist ein Ursachen-Wirkungs-Diagramm zur

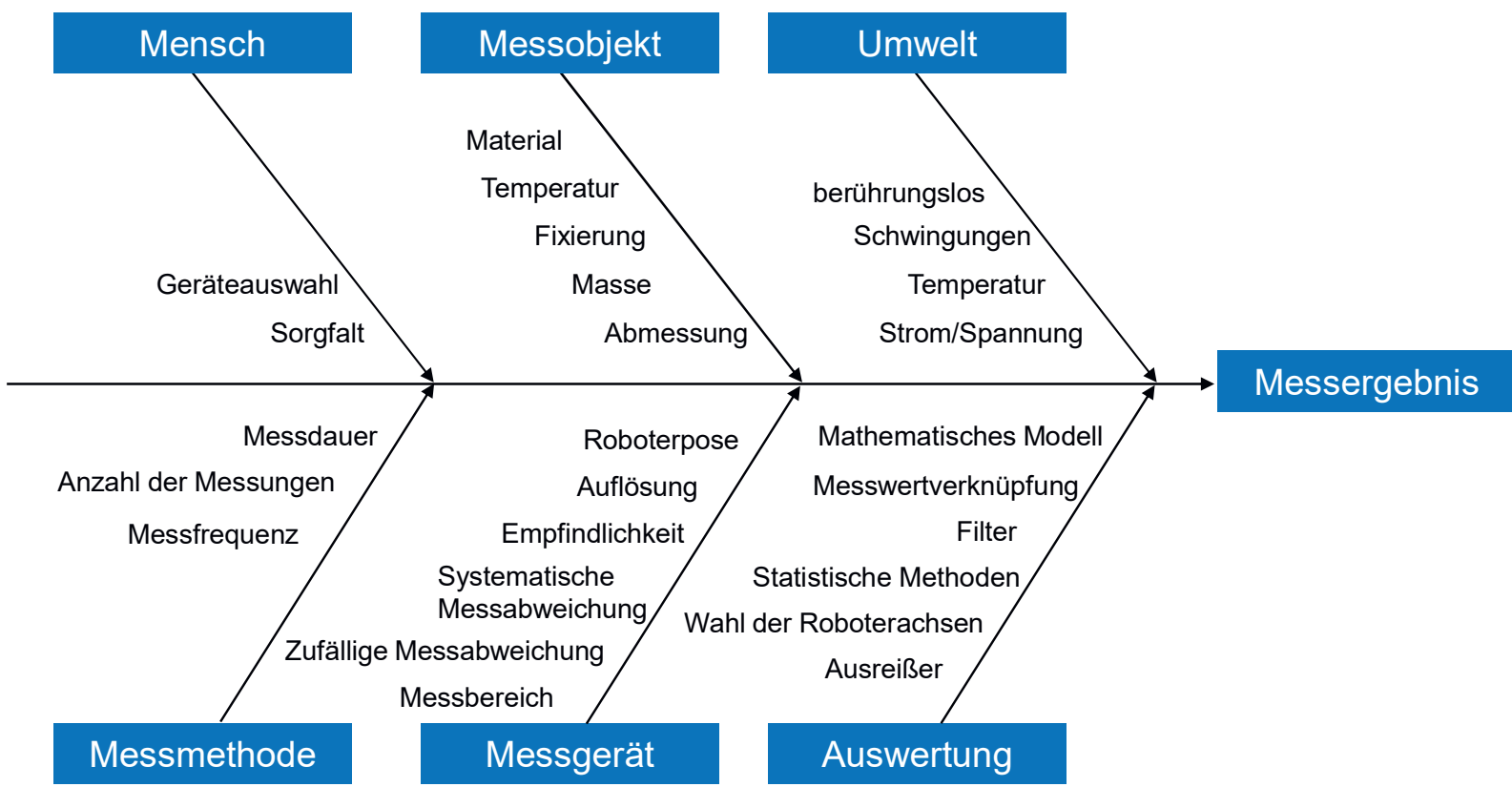

systematischen Suche nach den Ursachen [12]. Es zeigt Einflüsse auf das Messergebnis durch die Messmethode, Messgerät, Auswertung, Mensch, Messobjekt und die Umwelt auf. Die Messmethode beeinflusst beispielsweise das Messergebnis, durch die Dauer und Anzahl der Messungen sowie die Messfrequenz. Durch Filter, Messwertverknüpfungen oder Wahl der Daten einer spezifischen Roboterachse kann die Auswertung ebenfalls das Messergebnis verändern. Natürlich ist die Umwelt mit der Temperatur oder Schwingungen nicht zu vernachlässigen. Ebenso ist der Mensch ein Einflussfaktor, da die Wahl des Roboters und die Sorgfalt der Durchführung eine Auswirkung hat. Diese gesamten Einflüsse auf das Messergebnis wirken sich meistens als zufällige Abweichungen auf das Messergebnis aus.

Die eigentliche Messdurchführung am Roboter erfolgt in der Measure-Phase. Das Drehmoment (M) setzt sich dabei aus der Gravitationskraft (F) der gewählten Last und der Länge des Hebelarms ( $r$ ), d.h. von der Achse zum Roboterflansch, zusammen (GI.1).

$$
M=r \cdot F
$$

Bei der Analyze-Phase erfolgt das Aufbereiten, Auswerten und Visualisieren der Daten, um schlussendlich Wissen zu generieren. Bei der Betrachtung der Daten zeigt sich, dass durch die Anordnung der Achsen in der gewählten Pose im Messaufbau ein Hebelarm auf die Achsen 2, 4 und 6 entstehen und diese als sinnvolle Messergebnisse verwendet werden können. Auf den Achsen 1, 3, 5 und 7 wirkt hingegen kaum ein messbares Drehmoment.

\section{Abb. 4: Ursachen-Wirkungs-Diagramm der Einflüsse auf das Messergebnis}


Nachfolgend werden einige Ergebnisse vorgestellt:

- $\quad$ systematische Messabweichung

- zufällige Messabweichung

- Reproduzierbarkeit

- Empfindlichkeit

Die systematische Messabweichung, auch Bias genannt, ist die Abweichung zwischen dem Mittelwert der gemessenen Werte bei wiederholtem Messen $\left(\bar{x}_{g}\right)$ und dem Referenzwert des Merkmals $\left(x_{m}\right)$ (GI.2) [13].

$$
B i=\left|\bar{x}_{g}-x_{m}\right|
$$

In der Achse 2 zeigt sich ohne Last ein Drehmoment von $82,10 \mathrm{Nm}$. Bei einer zusätzlichen Last von $10 \mathrm{~kg}$ am Roboterflansch ergibt sich ein Drehmoment von 172,89 Nm. Die Drehmomentdifferenz liegt folglich bei 90,79 Nm. Der errechnete Referenzwert, aus Hebelarm multipliziert mit der Last ergibt annähernd den selben Wert. Daraus folgt ein Bias von $0,13 \mathrm{Nm}$. Um die Linearität der Drehmomentsensoren zu bestimmen, wurden Messungen mit <100 g, $500 \mathrm{~g}, 1 \mathrm{~kg}, 5 \mathrm{~kg}, 10 \mathrm{~kg}$ und $14 \mathrm{~kg}$ mit der Anzahl von $\mathrm{n}=25$ durchgeführt. Die Messdaten in Abb. 5 zeigen eine annährend lineare Kennlinie. Bei Gewichten unter $100 \mathrm{~g}$ ergibt sich ein max. Fehler von $15 \mathrm{~g}$. Es zeigt sich, dass ein geringer systematischer Messfehler vorliegt, der durch ein Offset ausgeglichen werden kann.

Für die zufällige Messabweichung wird die Standardabweichung verwendet. Sie ist das häufigst verwendete Maß um die Streubreite der Daten um den Mittelwert zu quantifizieren.

Die Standardabweichung der Messungen sind unter 0,03 Nm. Beispielsweise liegt in der Achse 2 ohne Last am Roboterflansch eine Standardabweichung von 0,018 Nm und bei einer Last von $14 \mathrm{~kg}$ eine Standardabweichung von $0,016 \mathrm{Nm}$ vor.

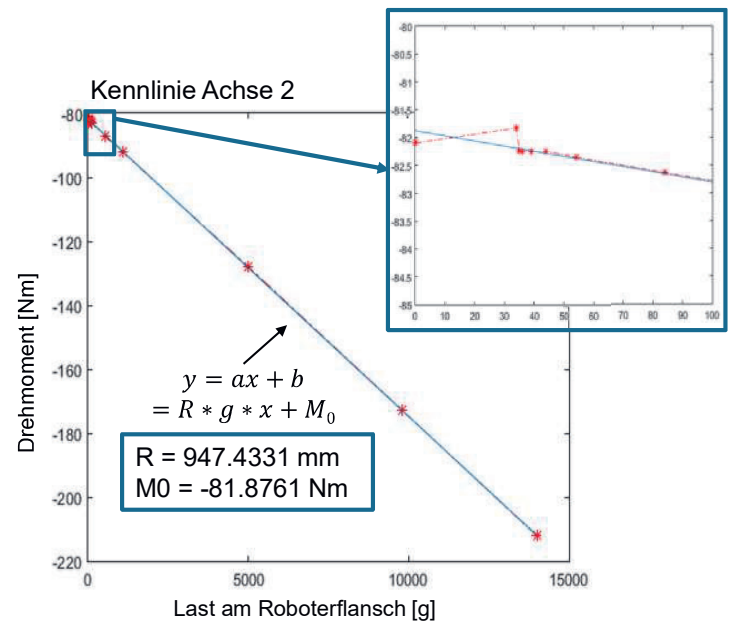

Drehmomentenverlauf Achse 2 Last $14 \mathrm{~kg}$

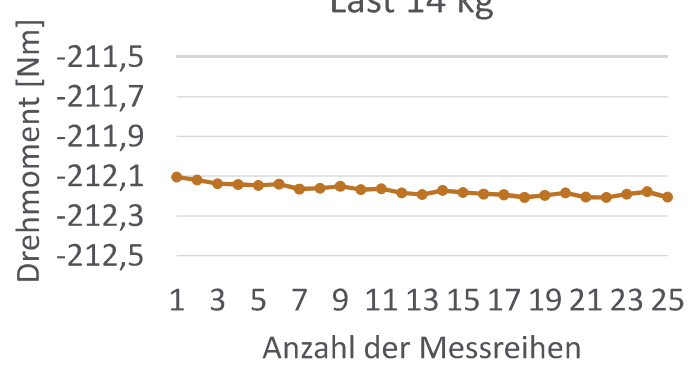

Abb. 6: Drehmoment in Achse 2 bei 25 Messungen bei einer Last von $14 \mathrm{~kg}$

Die Reproduzierbarkeit ist definiert als die Variation des Mittelwertes bei Messungen [7]. Bei dem wiederholten Messen mit einer Anzahl von $n=25$ unter denselben Messbedingungen, zeigen sich verschiedene Mittelwerte. Die Mittelwerte zeigen eine max. Drehmomentendifferenz von 0,10 Nm. In Abb. 6 ist der Verlauf des Drehmomentes bei 25 Messungen für die Last von $14 \mathrm{~kg}$ dargestellt. Des Weiteren zeigte sich, dass die Standardabweichung der Mittelwerte nach 25 Messungen konstant verläuft.

Für die Empfindlichkeit, d.h. den Einsatz des Roboters bei geringen Lasten zu bestimmen, wurden Gewichte von $5 \mathrm{~g}, 10 \mathrm{~g}, 20 \mathrm{~g}$ und $50 \mathrm{~g}$ am Flansch befestigt. Die Messungen wurden mit einer Anzahl $n=25$ durchgeführt. Es zeigt sich, dass die geringen Lasten in den Daten zu erkennen ist. Dennoch ist in Abb. 7 abzulesen, dass Lasten unter $20 \mathrm{~g}$ nicht differenziert werden können. Die Streuung der Messdaten ist zu hoch und lässt keine Unterscheidung zu. In Achse 2, 4 und 6 können ab $20 \mathrm{~g}$ eine nachvollziehbare Aussage getroffen werden.

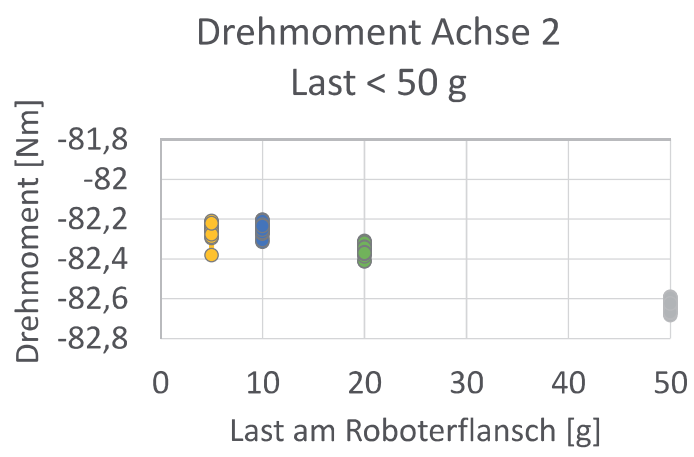

Abb. 7: Drehmoment in Achse 2 bei 25 Messungen mit geringen Lasten

Abb. 5: Linearität in Achse 2 
Grundsätzlich ist zwischen den sieben Roboterachsen zu unterscheiden. Achse 2 ist mit einem größeren Drehmomentsensor ausgestattet als beispielsweise Achse 7. Zu beachten ist, dass abhängig von der Pose, ein Hebelarm auf bestimmte Achsen wirkt. Dieser Hebelarm liefert Messdaten, die genutzt werden können. Die Messdaten haben eine zufällige Messabweichung, die durch verschiedene Faktoren beeinflusst werden. Die Messwerte streuen mit einer geringen Standardabweichung um den Mittelwert. Die systematische Messabweichung ist dahingegen gering. Eine lineare Kennlinie bei Messungen von verschiedenen Lasten ist gegeben, jedoch existieren geringe Abweichungen. In den Versuchen konnten Lasten ab $20 \mathrm{~g}$ detektiert werden.

\section{Diskussion}

Auf Basis dieser Ergebnisse können in der Improve-Phase zum einen die Verbesserung der Messergebnisse erfolgen und zum anderen eine konkrete Einordnung dieser Applikation in die Montage erfolgen.

Das Messergebnis kann in einem gewissen Rahmen verbessert werden. Dazu wird das Ishikawa-Diagramm (Abb. 4) aus der DefinePhase verwendet. Die hier beschriebenen Einflüsse können zum Teil reduziert werden, z.B. durch das Filtern der Daten. Ein gleitender Mittelwert ist eine Methode zur Glättung der Datenreihe und somit zur Reduktion der Streuung. Auch durch die Wahl der entsprechenden Roboterachse, z.B. nur Achse 2 oder Achse 2 und Achse 4, kann das Messergebnis verbessert werden.

Nachdem eine Betrachtung der Fähigkeiten des Roboters als Messgerät erfolgte, ist im nächsten Schritt das Konkretisieren der Anwendung in der Montage notwendig. Die Grundidee ist, die In-Prozess-Messung möglichst schlank („lean“), d.h. ohne zusätzliche Betriebsmittel, zu realisieren. Die allgemeinen Aufgaben im Montageprozess sind Handhaben, Fügen, Inbetriebnahme (bspw. durch Funktionsprüfung), Hilfsprozesse (bspw. durch Kontrollieren) und Sonderoperationen (bspw. durch Verpacken) [14]. Durch den Roboter können diese Aufgaben, vor allem der Hilfsprozess „Kontrollieren“, übernommen werden, auch vor dem Hintergrund, dass dieser Prozess in der Montage nicht wertschöpfend ist. Unter Kontrollieren gehört laut der VDI Norm 2860 unter anderem das Prüfen von Gewicht. In Form eines Ist- und Sollabgleiches von der Masse und Massenschwerpunkt (XYZ) können durch den Roboter Abweichungen ermittelt werden. Beispielhaft können bei Montagebeginn durch die integrierte Sensorik die statische Identifikation der Werkzeugdaten eines Robotergreifers erfolgen. Ebenso ist ein Gewichtsabgleich der Bauteile bei variantenhohen Produktionen vorstellbar, um somit eine Verwechselung auszuschließen.

In der Control-Phase erfolgen zum einen die Dokumentation der bisherigen Erkenntnisse sowie die Validierung. Dies bedeutet die Untersuchung im konkreten Anwendungsfall an einem Normal und eine vollständige Prüfprozesseignung durchzuführen. Diese Validierung ist Grundlage für die spätere Implementierung in der Montage.

\section{Zusammenfassung und Ausblick}

Ziel war es den Roboter als Messmittel zu klassifizieren und herauszufinden mit welcher Genauigkeit dieser eingesetzt werden kann. Mit dem Roboter soll das Prüfen von Gewicht und Massenschwerpunkt eines Objektes in der Montage erfolgen. Das Objekt kann dabei ein Werkzeug, zum Beispiel ein Greifer, oder ein Bauteil sein. Zur strukturierten Vorgehensweise wurden die DMAIC-Phasen (Define, Measure, Analyze, Improve, Control) aus dem Qualitätsmanagement für die Untersuchung des Roboters verwendet. Ausgefüllt wurden die Phasen mit den Ansätzen aus der Prüfprozesseignung. Diese neu entwickelte Methodik dient dazu einen Roboter als Messgerät zu untersuchen und kann ebenfalls auf andere Anwendungsfälle übertragen werden. Mithilfe eines Ishikawa-Diagramms konnten zuvor Einflüsse auf das Messergebnis ermittelt werden. Die Messungen wurden in einer Roboterpose über die gesamte Nutzlast durchgeführt, so dass Wissen über die systematische und die zufällige Messabweichung generiert wurde.

Die Untersuchung mit der entwickelten Vorgehensweise zeigte einen Vertrauensbereich für den Roboter auf. Im nächsten Schritt erfolgt die Validierung der Erkenntnisse an einem konkreten Anwendungsfall. Dazu zählt ein praxistauglicher Messablauf mit optimierter Messdauer, Bestimmung der Roboterachsen als Messwertgeber, Filterung der Daten usw. um schlussendlich die gesamte Messunsicherheit zu bestimmen. 


\section{Danksagung}

Dieser Beitrag entstand im Rahmen des Forschungsprojektes „messtechnisch gestützte Montage", welches aus Mitteln des Europäischen Fonds für regionale Entwicklung (EFRE) gefördert wird.

\section{Literaturnachweis}

[1] VDI/VDE-Gesellschaft Mess- und

Automatisierungstechnik (GMA),

Fertigungsmesstechnik 2020 - Technologie-

Roadmap für die Messtechnik in der industriellen Produktion, Düsseldorf, (2011).

[2] CP. Keferstein, M. Marxer, C. Bach,

Fertigungsmesstechnik, Springer Vieweg, Wiesbaden,. 279-298 (2018); doi: 10.1007/9783-658-17756-0-6

[3] T. Ohno, W. Hof, E. Stotko, Das ToyotaProduktionssystem: das Standardwerk zur Lean Production, 3. Auflage, Campus-Verlag, Frankfurt am Main, (2013).

[4] BIPM, IEC, IFCC, ILAC, ISO, IUPAC, IUPAP, OIML, Evaluation of measurement data - An Introduction to the „Guide to the expression of uncertainty in measurement" and related documents, Joint Committee for Guides in Metrology, JCGM 104:2009, (2009).

[5] BIPM, IEC, IFCC, ILAC, ISO, IUPAC, IUPAP, OIML, International vocabulary of metrology Basic and general concepts and associated terms (VIM), Joint Committee for Guides in Metrology, JCGM 200:2008, 3. Auflage, (2012).

[6] Verband der deutschen Automobilindustrie e. V. (VDA), Prüfprozesseignung - Eignung von Messsystemen, Mess- und Prüfprozessen, Erweiterte Messunsicherheit, Konformitätsbewertung, Band 5, Frankfurt/M., 2. Auflage, (2011).

[7] A.I.A.G. - Chrysler Group LLC, Ford Motor Company, General Motors Corporation, Measurement System Analysis (MSA) Reference Manual, 4. Auflage, Michigan, (2010).

[8] E. Dietrich, Wo liegen die Unterschiede?: Vergleich von MSA und VDA Band 5. QZ Qualität und Zuverlässigkeit, 30-34 (2011).

[9] T. Bauernhansl, M. ten Hompel, B. VogelHeuser, Industrie 4.0 in Produktion, Automatisierung und Logistik: Anwendungen, Technologien, Migration, Springer Fachmedien Wiesbaden, (2014); doi: 10.1007/978-3-65804682-8

[10] KUKA Roboter GmbH, Sensitive Robotik_LBR iiwa. https://www.kuka.com/de-de/produkteleistungen/robotersysteme/industrieroboter/lbriiwa, (2016).

[11] ML. George, D. Rowlands, M. Price, J. Maxey, Das Lean Six Sigma Toolbook: Mehr als 100 Werkzeuge zur Verbesserung der Prozessgeschwindigkeit und -qualität, 1. Auflage., Verlag Franz Vahlen, (2016).

[12] W. Geiger, W. Kotte, Handbuch Qualität: Grundlagen und Elemente des Qualitätsmanagements: Systeme, Perspektiven, 5. Auflage, Vieweg \& Teubner
Verlag, Wiesbaden, (2008); doi: 10.1007/978-38348-9429-8

[13] E. Dietrich, Leitfaden zum „Fähigkeitsnachweis von Messsystemen“, Birkenau, Version 2.1, (2002).

[14] G. Pahl, W. Beitz, J. Feldhusen, K-H. Grote, Konstruktionslehre: Grundlagen erfolgreicher Produktentwicklung: Methoden und Anwendung, 7. Auflage, Springer Vieweg, Heidelberg, (2007); doi: 10.1007/978-3-54034061-4 